\title{
A Rare Case of Superior Mesenteric Vein Aneurysm Secondary to Portal Hypertension
}

\author{
Anjana V. Trivedi, Maulik C. Jethva, Malek Mohmed Anwar, Chetna K. Dodia, Jagruti G. Kalola
}

Radiology Department, PDU Medical College \& Sanya GIC Imaging Centre, Rajkot, India.

Email: doctorjitendra@yahoo.com

Received November $11^{\text {th }}, 2012$; revised December $28^{\text {th }}, 2012$; accepted January $9^{\text {th }}, 2013$

\begin{abstract}
Venous aneurysms are less common than arterial aneurysms in clinical practice, and the occurrence of such cases is a topic for publication. Aneurysms of the superior mesenteric vein (SMV) are rare, and their origin is unknown. Many aneurysms are asymptomatic, and the diagnosis is established from radiologic findings. Others are diagnosed after complications such as gastrointestinal bleeding or thrombosis with associated abdominal pain. Because of the rarity of this disease, therapy must be adapted to fit each case. A 55-year-old woman presented with pain in abdomen since 2 months. The diagnosis of this anomaly was made after Computed tomography (CT) scans, that demonstrated a mass. Apart from various etiological theories, portal hypertension was more likely cause of her SMV aneurysm. Case was treated by medical treatment like Sorbitrate, Propranolol, Omeprazole and Lasix. No aneurysm growth or complication was observed even after 2 years of follow-up.
\end{abstract}

Keywords: Venous Aneurysm; Superior Mesenteric Vein (SMV); CT Scan

\section{Introduction}

Venous aneurysms are less common than arterial aneurysms in clinical practice, and the occurrence of isolated cases is a topic of publication. Aneurysms of superior mesenteric vein are rare. The etiology of this aneurysm is unclear, as they are found in both asymptomatic individuals and in those with concurrent disease. Here, we present a case with no predisposing factor.

\section{Case Report}

A 55-year-old woman presented with pain in abdomen since last 2 months. There was a history of one time hematemesis. This was not accompanied by any alteration in intestinal habits, increase in abdominal volume or weight loss. There was no history of abdominal trauma.

Physical examination revealed good general health. Chest auscultation was normal. Clinically, the abdomen appeared normal except enlarged spleen. Vascular physiccal examination demonstrated normal arterial pulses and absence of edema. There was no evidence of collateral venous circulation in the abdomen.

To find out the cause of pain in abdomen, Sonography of abdomen was done, which showed splenomegaly and dilated portal vein with possibility of aneurismal dilatation of SMV. Splenomegaly was due to high back pressure in splenic vein because of portal hypertension. Liver and other structures were normal. There was no finding of cirrhosis of liver. For further evaluation, computed tomography was performed, which showed SMV was dilated to $3.5 \mathrm{~cm}$, without internal thrombus (Figure 1) near the level of the portal confluence. With contrast study, opacification of SMV was clearly seen (Figure 2). To rule out oesophageal varices, oesophagoscopy was done which showed oesophageal varices. As our patient had no serious complaint regarding SMV aneurysm and there was no thrombus inside the aneurysm, surgical intervention was not done and case was treated by medical line of treatment. Tab Sorbitrate, Tab Propranolol, Tab Omeprazole and Tab. Lasix were given. Later on splenectomy was done. Patient was called regularly for follow-up and ultrasonography was done at regular interval. The size of aneurysm was stable and there was no complication even 2 years after the diagnosis of SMV aneurysm.

\section{Discussion}

At the level of pancreatic head, the portal vein is formed by the confluence of the superior mesenteric vein (SMV) and splenic veins. It extends into the hepato-duodenal ligament and is divided into the right and left branches to supply both lobes of the liver.

The SMV is located anteriorly and to the right side of the superior mesenteric artery and postero-medial to the 
head of pancreas. Normally, the SMV measures $1.2 \mathrm{~cm}$ in diameter [1]. Its size was reported as abnormal if it measured more then $1.6 \mathrm{~cm}$ in diameter.

SMV aneurysm is a rare condition; the first case was reported by Barzilai and Kleckner in 1956 [2]. The origin of this aneurysm is unknown, but some theories have been put forward. The exact pathogenesis behind the formation of these aneurysms is still not completely understood, yet arguments exist to support both, congenital and acquired mechanisms. Weakness of the vessel wall may give rise to venous dilatation, even under normal venous pressure [3] or as a result of portal hypertension. Another possibility involves an embryologic mechanism; During embryonic development, three anastomoses form between right and left vitelline veins around the future duodenum.

A complex process of involution and interconnection of these vitelline veins results in the portal vein. Abnormal development of the portal venous system during this critical period may give rise to an extrahepatic portal vein aneurysm. Incomplete regression of the distal right primitive vitelline vein, or a variant branching pattern of the portal vein may later form a portal vein aneurysm. Incomplete regression of the distal right vitelline vein leads to a diverticulum that would develop into an aneurysm in the proximal superior mesenteric vein [3]. A third possible etiology could be the weakening of the

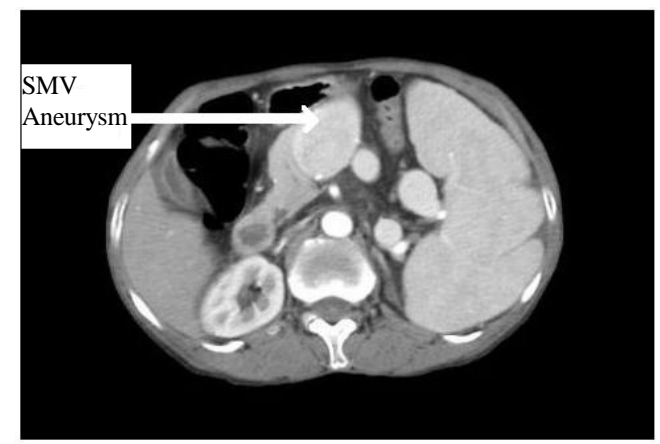

Figure 1. Axial section of CT scan shows a dilated $(3.5 \mathrm{~cm}$ AP diameter) superior mesenteric vein without internal thrombosis.

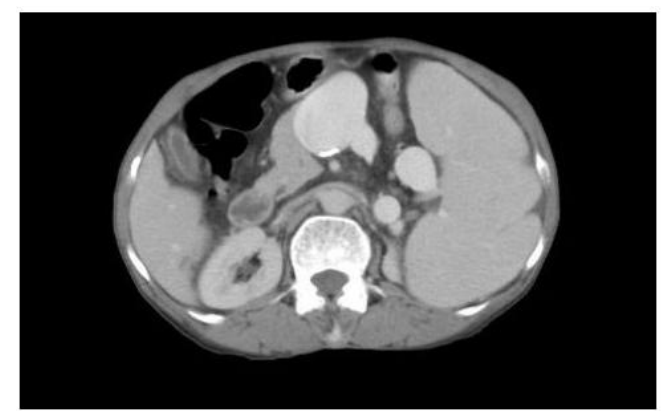

Figure 2. Axial section of CT scan (after contrast) shows a dilated (3.5 cm AP diameter) superior mesenteric vein. vessel wall with concomitant dilatation by enzymatic digestion occurring during pancreatitis. Our patient did not have such history but have portal hypertension suggesting most probable cause of SMV aneurysm.

In clinical practice, venous aneurysms are not common; most patients with intra-abdominal venous aneurysm seek medical assistance because of vague abdominal pain. Some patients may have gastrointestinal bleeding, acute venous occlusion, or pulmonary embolism [4].

In most cases reported, sonography was the first imaging technique performed, because it is noninvasive. The aneurysm appeared as an anechnoic structure near the head of pancreas. Their vascular nature is confirmed with color Doppler. Color Doppler sonography reveals that these aneurysms fill completely with color flow unless they contain thrombosis. Duplex Doppler sonography demonstrates a monophasic wave form characteristic of the superior mesenteric vein. Flow within the superior mesenteric vein is directed toward the portal confluence.

CT scan reveal the size and extent of the lesion, and confirms its vascular origin, but requires use of iodinated intravenous contrast medium [5]. Venous phase mesenteric angiography can be performed to confirm the diagnosis [6]. The multiplaner capability of MRI along with the capacity to render angiogram like images of vascular structures make MRI well suited to the evaluation of such an aneurysm.

However, due to invasiveness of angiographic procedure, sonography, CT and MRI are preferred in most instances.

Since the number of cases of SMV aneurysm is small, the natural history and clinical evolution of such aneurysms are not clear.

Management ranged from watchful waiting to intervention. In most cases, the indication for surgery was the occurrence of a complication (thrombosis and rupture) or the presence of symptoms. Operations performed included splenectomy, aneurysmorrhaphy, aneurysmectomy, aneurysmectomy and splenectomy, aneurysmectomy with splenectomy and shunt, aneurysmorrhaphy and splenectomy, distal pancreatectomy and splenectomy, aneurysmorrhaphy and portocaval shunt, splenectomy, distal pancreatectomy and lienorenal shunt, splenectomy and splenorenal shunt, portocaval shunt, splenorenal shunt and transhepatic thrombectomy and thrombolysis [7]. Initially it was thought that surgical intervention with either shunting or aneurysmorrhaphy procedure was the treatment of choice, particularly in cases with evidence of portal hypertension [8]. First line management has changed over time, with there being a shift from surgical to conservative management.

However, instead of the surgical approach, it may now be appropriate to follow up patients conservatively with 
serial imaging [9]. Because an SMV aneurysm is a rare anomaly and its long-term evolution is not known, patients should be clearly informed of possible complications, including rupture and thrombosis.

\section{REFERENCES}

[1] L. Bolondi, L. Gandolfi and V. Arienti, "Ultrasonography in Diagnosis of Portal Hypertension," Radiology, Vol. 142, No. 1, 1982, pp. 167-172.

[2] R. Barzilai and M. S. Kleckner, "Hemocholecyst Following Ruptured Aneurysm of Portal Vein,” Archives of Surgery, Vol. 72, No. 4, 1956, pp. 725-727. doi:10.1001/archsurg.1956.01270220173023

[3] H. Schild, F. Schweden and B. Braun, "Aneurysm of the Superior Mesenteric Vein,” Radiology, Vol. 145, No. 3, 1982, pp. 641-642.

[4] K. Nishinari, N. Wolosker, G. Yazbek, W. T. Nakagawa and A. Lopes, "Idiopathic Aneurysm of Inferior Vena Cava Associated with Retroperitoneal Ganglioneuroma:
Case Report,” Journal of Vascular Surgery, Vol. 37 No. 4, 2003, pp. 895-898. doi:10.1067/mva.2003.172

[5] J. Seda, B. Padovani and S. Chanalet, "Aneurysm of the Superior Mesenteric Vein,” American Journal of Roentgenology, Vol. 161 No. 1, 1993, pp. 903-904.

[6] A. Fulcher and M. Turner, "Aneurysms of the Portal Vein and Superior Mesenteric Vein,” Adominal Imaging, Vol. 22, No. 3, 1997, pp. 287-292. doi:10.1007/s002619900191

[7] D.Athanasios Giannoukas, S. Giorgos Sfyroeras. "Current Management of Visceral Venous Aneurysm,” Phlebolymphology, Vol. 17 No. 3, 2010, pp. 130-136.

[8] W. L. Perret and A. de Silva, "Portal Circulation Aneurysms: Two Cases Reviews,” Australasian Radiology, Vol. 51 No. 1, 2007, pp. 87-90. doi:10.1111/j.1440-1673.2006.01666.x

[9] A. Fulcher and M. Tumer, "Aneurysm of the Portal Vein and Superior Mesenteric Vein,” Abdom Imaging, Vol. 22, No. 3, 1997, pp. 287-289. doi:10.1007/s002619900191 